\title{
Presentación del monográfico Educación inclusiva: avances desde la reflexión, la práctica y la investigación
}

\author{
Coordinado por Alejandro Rodríguez-Martín y Emilio Álvarez-Arregui
}

\section{La educación o es inclusiva o no es educación}

Estas palabras que convertimos en frase categórica y que pudiera contener -aunque consideramos que no- algún toque redundante para comenzar esta presentación tiene, precisamente, un objetivo claro de animar e invitar a la lectura de este monográfico pero, también, de incitar a la reflexión junto a dos términos que no debemos ni podemos, necesariamente, desligar del término "educación": calidad y equidad.

Se trata, desde el primer momento, de suscitar en quienes se acerquen a esta lectura tres interrogantes de gran calado sobre la educación inclusiva: cómo la entendemos, qué hacemos para lograrla y cómo podemos seguir mejorando nuestras prácticas y comunidades educativas para construir, desarrollar y consolidar entre todas y todos, un verdadero sistema educativo que sitúe el derecho primordial a la diversidad como un eje vertebrador del cambio en los centros educativos.

A nadie se le escapa a estas alturas que existe, en comparación con décadas pasadas, un mayor compromiso hacia la educación de todas las personas por parte de las administraciones públicas, al menos en nuestro país y entorno cultural. La Educación para todas las personas constituye un compromiso y una responsabilidad mundial para ofrecer una educación de calidad para todas y todos; adaptada a la diversidad de personas y contextos y a lo largo de toda la vida. Esta visión amplia del aprendizaje, iniciada en la Conferencia Mundial sobre Educación para Todos de Jomtien (Tailandia), en 1990, bajo el auspicio de la UNESCO ha ido, progresivamente, cristalizando en desarrollos legales. En este sentido, son prolijas -en nuestro país y en nuestro entorno europeo- numerosas leyes, declaraciones, recomendaciones, etc. que sitúan -al menos en el plano formal- la educación inclusiva como referente y como derecho.

A este respecto podríamos destacar, aun a riesgo de equivocarnos por la frecuencia y celeridad con la que la Unión Europea acuerda y difunde propuestas, el último Dictamen de Comité Económico y Social Europeo sobre "Una educación de alta calidad para todos" (Diario Oficial de la Unión Europea de 31 de mayo de 2017) donde se insta a los Estados miembros a adoptar un compromiso más firme en el ámbito de la educación de calidad para todas las personas con el fin de cumplir los objetivos de la Unión Europea para 2020 y de las Naciones Unidas para 2030. También se subraya la importancia de que la educación y la formación de calidad para todas y todos cuente con financiación pública, a la vez que destaca la conveniencia de educar a la ciudadanía en los derechos humanos y en reforzar y consolidar los valores europeos en todos los programas educativos de los Estados miembros.

Precisamente los valores son, a nuestro entender, el núcleo de lo que hemos venido denominando "Educación inclusiva", un conjunto de valores y principios éticos ya expuestos por Booth (2006) y por Ainscow $(2009,2016)$ y ambos autores conjuntamente_(Booth y Ainscow (2002) y que tiene un importante impacto en el proyecto de sociedad que deseamos construir. Hablamos, pues, de la diversidad humana, de la biodiversidad del planeta y su protección, la participación, los derechos o el cuidado de los más vulnerables, entre otros. Precisamente estos valores están sufriendo importantes envites ya que, durante los últimos años, y especialmente en 2016 y los meses que han trascurrido en este año 2017, hemos asistido a contextos de violencia de diversos orígenes como por ejemplo el terrorismo que utiliza argumentos religiosos fundamentalistas; el fuerte rechazo de las personas refugiadas que huyen de la guerra o el conflicto; o los grupos políticos de extrema derecha que defienden propuestas xenófobas y racistas.

Ante este escenario, se hace más importante que nunca -pese a lo discutible de las prácticas- los valores sobre los que reside nuestro entorno sociocultural y, en esta línea, destacar la Declaración sobre la promoción de la ciudadanía y de los valores comunes de libertad, tolerancia y no discriminación a través de la educación, firmada por los ministros de Educación en París en marzo de 2015 tras los ataques cometidos en Francia y Dinamarca, y donde se plasma la determinación de trabajar por los valores fundamentales que constituyen el núcleo de la Unión: el respeto de la dignidad humana, la libertad (incluida la libertad de expresión), la democracia, la igualdad, el Estado de Derecho y el respeto de los derechos humanos.

Nos encontramos, por tanto, ante un panorama internacional bastante convulso y tensionado por diversas circunstancias que debería tener repercusiones directas -desde planteamientos de cambio y mejora en el sistema educativo-. Pero ello requiere adoptar una perspectiva compartida, trasversal y corresponsable de la inclusión en su sentido más amplio alejándonos de reduccionismos simplistas vinculados sólo a situaciones de discapacidad o colectivos en riesgo de exclusión. Implica una aspiración más alta, arriesgada y noble: una educación democrática (Escudero y Martínez, 2012). En otras palabras, se trata de la promoción del aprendizaje, el fomento de la participación y de la lucha contra la discriminación, como tareas que nunca terminas y que nos involucran a todas y todos en la reflexión y en la reducción de las barreras al aprendizaje y la participación que hemos y seguimos creando y que generan fracaso escolar y marginación (Booth y Ainscow, 2015, p. 9). En definitiva, supone un compromiso claro con la equidad educativa: acoger a todo el alumnado y hacerles partícipes de verdad.

Parar lograr tales compromisos la educación debe superar importantes desafíos, más aún después de la devastadora crisis 
económica que hemos experimentado en nuestro entorno y que, de manera especial, ha afectado drásticamente a los recursos humanos y materiales del sistema educativo y, lo que es más importante, cómo los recortes han afectado de manera difícilmente reparable, a la emoción y la ilusión del conjunto de la comunidad educativa. A esto, además, debemos añadir -porque no podíamos obviarlo bajo ningún concepto- la necesaria estabilidad legal que todo sistema educativo reclama.

Desde estos referentes, el monográfico presentado desea e invita a repensar la educación inclusiva desde su conceptualización hasta su desarrollo en experiencias concretas y abordando todas las etapas educativas, desde la Educación Infantil a la Universidad. Se pretende recoger y divulgar reflexiones e investigaciones que muestren un aporte de interés para el campo educativo avanzando en su configuración en las tres dimensiones que, de manera, tangencial, lo abordan de acuerdo con el Index for Inclusion: culturas, políticas y prácticas. Por ello, dado la diversidad de dimensiones, estudios y análisis posibles, es por lo que se ha optado en confeccionar esta monografía con tres grandes bloques que, posteriormente detallaremos y que, en suma, abordan la educación inclusiva desde un plano reflexivo, desde la perspectiva del profesorado y desde las experiencias con el alumnado en todas las etapas de nuestro sistema educativo.

Iniciamos este monográfico con dos reflexiones de máximo nivel y de carácter internacional que nos presentan Francesco Tonucci, Tony Booth y Zafra Margolin.

En el primer caso, Tonucci (Instituto de Ciencias y Tecnologías de la Cognición), famoso pedagogo y dibujante italiano que además ha sido nombrado Doctor Honoris Causa por la Universidad de Oviedo, plantea un rico, provocador e interesante análisis sobre la diversidad como valor en una escuela que cambia. Para ello hace un recorrido por la finalidad clave de la escuela que es el desarrollo integral de la persona y, por supuesto tratándose de Tonucci y su alter ego FRATO, hace una defensa cerrada e indiscutible del protagonismo de los niños y las niñas, de su derecho a la libertad de expresión y de la importancia de abordar todos los lenguajes en las aulas, especialmente los lenguajes de carácter artístico. Tonucci plantea, también, la necesaria reforma metodológica y organizativa de las aulas para concluir abordando la importancia de la formación del profesorado para el logro de tan importantes retos.

La aportación que realiza la profesora Zafra Margolin (Malta Conference Foundation) se centra en hacer accesible a todas las personas la enseñanza de la ciencia y, para ello, plantea métodos didácticos creativos que permitan la retención del talento a todos los niveles. Estos procedimientos didácticos que se plantean utilizan todos los lenguajes posibles al alcance de las personas y tienen un núcleo común en la participación conjunta de profesorado, familia y alumnado.

La siguiente aportación la realiza el profesor Gerardo Echeita (Universidad Autónoma de Madrid y Consorcio para la Educación Inclusiva) que plantea un análisis del significado global que tiene el derecho a una educación inclusiva, profundizando en el sentido, las dimensiones, y dilemas a los que hoy se enfrentan los agentes educativos encargados de implementarla, en particular en España, si bien muchos de ellos son similares en otras partes del mundo. La contradicción fragrante entre lo que se dice en las normas y lo que realmente ocurre en muchos centros educativos genera enormes tensiones en toda la comunidad educativa.

Continuando con el análisis sobre el alcance y desafíos de la educación inclusiva, las profesoras Begoña Vigo y Belén Dieste (Universidad de Zaragoza) presentan un interesante estudio, realizado desde un proyecto etnográfico multiescalar, que intenta mostrar la comprensión que unos conjuntos de escuelas tienen sobre sí mismas. El conocimiento del lugar y espacio, la conexión de la vida del alumnado con el curriculum y el desarrollo pro- fesional del profesorado son estrategias que dan cuenta de las prácticas de educación inclusiva en la escuela.

Tras este bloque inicial, de reflexión e investigación sobre el alcance de la educación inclusiva, comenzamos un apartado de investigaciones referidas a la figura del profesorado desde diferentes dimensiones, consideraciones y contextos. Así, las profesoras Francisca González-Gil, Elena Martín-Pastor y Begoña Orgaz Baz (Universidad de Salamanca), presentan la validación de una escala para evaluar la formación inicial en educación inclusiva de estudiantes universitarios de las titulaciones de educación. Por su parte, Dolors Forteza y Francisca Moreno (Universitat de les Illes Balears) analizan los procesos que obstaculizan la inclusión en la educación secundaria obligatoria poniendo de manifiesto la necesidad de la formación del profesorado y el desarrollo de respuestas educativas significativamente contextualizadas para favorecer la inclusión de todo el alumnado.

Dentro de este apartado de investigaciones centradas en el profesorado, hay una interesante aportación sobre el análisis las funciones, tareas y actuaciones del profesorado de apoyo en las prácticas inclusivas de aula en centros ordinarios. La investigación, obra de los profesores Jesús Soldevila, Mila Naranjo y Joan Jordi Muntaner (Universitat de Vic y Universitat de les Illes Balears) plantean una investigación de carácter longitudinal fundamentada en tres ejes: el concepto de educación inclusiva, la determinación de buenas prácticas para la inclusión y el rol del profesor de apoyo. Los resultados han permitido definir diferentes prácticas inclusivas relacionadas con el profesorado de apoyo, que repercuten en un incremento significativo de la presencia, participación y progreso del alumnado con diversidad funcional en el aula ordinaria.

Otra experiencia vinculada a la figura docente, la presentan Manuel Cotrina, Mayka García y Ester Caparrós (Universidad de Cádiz) al abordar la "pareja pedagógica" como estrategia didáctica innovadora de corte inclusivo, que caracteriza una modalidad de enseñanza colaborativa (co-enseñanza). Esta estrategia requiere la implicación, intencional y voluntaria de dos profesores en todos los aspectos vinculados a la docencia: planificación, evaluación y, singularmente, durante el desarrollo de las sesiones de clases.

Por su parte, los profesores José Ma Fernández-Batanero, Pedro Román y Mohammed El Homrani (Universidad de Sevilla y Universidad de Granada) presentan los resultados obtenidos en una investigación cuyo objetivo ha sido identificar el nivel de formación y conocimiento tecnológico que el profesorado de educación primaria, de las provincias de Granada, Huelva, Jaén y Sevilla, tienen respecto a la aplicación de las TIC para personas con discapacidad. Como resultado más destacado está la necesidad de formación del profesorado en este aspecto.

Las profesoras Amaya Cepa, Davinia Heras y María Fernández-Hawrylak (universidad de Burgos), inician el último bloque del monográfico centrado en investigaciones concretas en contextos de aula de las diferentes etapas educativas. Su estudio se enmarcada dentro del ámbito de la Educación Emocional en edades tempranas y el objetivo ha sido comprobar la eficacia del Programa EMO-ACCIÓN de educación emocional, valorando su impacto en niños de 4 a 5 años con necesidades específicas de apoyo educativo. Los resultados obtenidos muestran que el alumnado incrementa de forma significativa sus competencias emocionales sin distinguir la existencia o no de necesidades específicas.

El siguiente estudio que se presenta lo realizan los profesores Rafael Santana, Jesús A. Alemán y Manuel López (Universidad de Las Palmas de Gran Canaria y Universitat de Valéncia) y está centrado en la necesidad de evidenciar a los niños y niñas pequeños qué hay en los libros y qué representan, mucho antes de que aprendan a leer. En su aportación recogen una revisión 
de investigaciones sobre los efectos y beneficios de la lectura en voz alta entre familiares o educadores/as infantiles y las niñas y niños desde edades muy tempranas, lo que constituye una estrategia que favorece la implementación más rápida y adelantada del aprendizaje formal del código escrito en las escuelas.

La participación del alumnado en proyectos educativos vinculados al territorio es la investigación planteada por los profesores José F. Amiama y Javier Monzón (Universidad del País Vasco/Euskal Herriko Unibertsitatea) y la profesora Nieves Ledesma (Universidad Pública de Navarra) y cuya hipótesis de partida es que la participación y la toma de decisiones del alumnado en trabajos vinculados con el contexto facilita la inclusión de todo el alumnado. En este estudio se aborda, desde la perspectiva de la inclusión, el efecto de las medidas ordinarias de atención a la diversidad adoptadas en el marco de un proyecto interdisciplinar realizado en $3^{\circ}$ ESO de acondicionamiento de un espacio escolar realizado por el alumnado recabando el apoyo de diversas entidades de su municipio. Los resultados muestran que se generan prácticas más inclusivas porque existe un verdadero compromiso con una tarea, hay vinculación con el territorio mejora el autoconcepto del alumnado y sus sentimientos de pertenencia a una colectividad.

Cerramos el monográfico con una investigación centrada en el ámbito universitario. Los profesores Emilio Álvarez-Arregui, Alejandro Rodríguez-Martín y Susana Agudo Prado (Universidad de Oviedo) con el apoyo del investigador Xavier Arreguit (Innobridge SA) presentan diferentes proyectos que se integran en un marco general que aborda la construcción, desarrollo e implementación de un modelo ecosistémico inclusivo que integra la formación, la investigación y la innovación desde el que gestionamos el conocimiento y las redes de instituciones, de profesionales y usuarios para dar respuestas integrales a las necesidades y demandas de las organizaciones con las que interactuamos y de la ciudadanía en general.

No queremos finalizar esta presentación del monográfico sin retomar, de nuevo, el inicio que hemos empleado al animar e invitar a su lectura desde un posicionamiento comprometido y crítico. Sólo resta indicar, por un lado, que las limitaciones espaciales han impedido incorporar y dar respuesta a otras investi- gaciones y propuestas, sin duda, de gran importancia; por ello confiamos en que la selección realizada sea del interés de la comunidad educativa y las administraciones, así como constituir un referente más para seguir investigando en estos contenidos. Por otro lado, queremos terminar con el reconocimiento público a todas las personas que han contribuido a realizar este monográfico por su generosidad para hacernos partícipes del conocimiento que se deriva de la calidad de los trabajos presentados asís como de los constantes apoyos que tenido para la edición, impresión y difusión de la Revista Aula Abierta.

Gracias a todas y todos y gracias a los miles de profesionales que día a día trabajan en las aulas de todas las etapas educativas creyendo que la diversidad es un valor y que son muchas más las cosas que nos unen de las que nos separan.

\section{Bibliografía}

Ainscow, M. (2009). Desarrollo de escuelas inclusivas. Madrid: Narcea

Ainscow, M. (2016). Struggles for Equity in Education. Londres. Routledge

Booth, T. \& Ainscow. M. (2002). Index for inclusión. Guía para la evaluación y mejora de la educación inclusiva. Madrid: Consorcio para la Educación Inclusiva.

Booth, T. (2006). Manteniendo el futuro con vida; convirtiendo los valores de la inclusión en acciones. En M.A. Verdugo y F.B. Jordán de Urríes (Coords.), Rompiendo inercias. Claves para avanzar. VI Jornadas Científicas de Investigación sobre Personas con Discapacidad (pp. 211-217). Salamanca: Amarú.

Booth, T. y Ainscow. M. (2015). Guía para la Educación Inclusiva. Desarrollando el aprendizaje y la participación en los centros escolares. Madrid: OEI/FUHEM

Dictamen de Comité Económico y Social Europeo sobre "Una educación de alta calidad para todos. Diario Oficial de la Unión Europea C 173 de 31 de mayo de 2017, p. 1-7.

Escudero, J. M. y Martínez, B. (2012). Las políticas de lucha contra el fracaso escolar: ¿programas especiales o cambios profundos del sistema y la educación? Revista de Educación, Ext., 174-193. 
